\title{
A Model-Driven Decision Support System for the Master Planning of Ceramic Supply Chains with Non-uniformity of Finished Goods
}

\author{
I. MUNDI ${ }^{1}$, M. M. E. ALEMANY', A. BOZA ${ }^{2}$, R. POLER ${ }^{2}$ \\ ${ }^{1}$ Escuela Técnica Superior de Ingenieros Industriales, \\ Universitat Politècnica de València, \\ Camí de Vera s/n, 46022 València, Espanya, \\ mamunsan@posgrado.upv.es \\ ${ }^{2}$ Centre d’Investigació de Gestió i Enginyeria de Producció (CIGIP), \\ Universitat Politècnica de València, \\ Camí de Vera s/n, 46022,València,Espanya, \\ 2 mareva@omp.upv.es, 3aboza@omp.upv.es,4rpoler@cigip.upv.es
}

Abstract: In this paper, a Model-Driven Decision Support System (DSS) for the Master Planning of ceramic Supply Chains characterized by producing units of the same finished good in a specific lot that differ in the aspect (quality), tone (colour) and/or gage (thickness) is proposed. The DSS is based on a mathematical programming model reflecting these non-uniformity characteristics. Through the different DSS functionalities, Decision Makers can generate different scenarios by means of changing any data. Optimal solution of each scenario can be evaluated for robustness under other scenarios. The Decision Maker can compare different solutions and finally choose the most satisfactory one for being implemented. To demonstrate the validity of the DSS, a realistic example is described through the generation of different scenarios based on the degree of finished goods uniformity in lots.

Keywords: Model-Driven Decision Support System, Master Planning, Ceramic Supply Chains, Lack of Uniformity

\section{Introduction ${ }^{1}$}

Supply chains (SCs) operations planning is a complicated task due to the existence of a huge number of decisions, constraints, objectives (sometimes conflictive), possible alternatives to be evaluated and the presence of uncertainties. For the case of ceramic SCs, this planning task becomes even more complex due to the appearance of the so called Lack of Homogeneity in the Product (LHP) [1].

LHP in ceramic SCs implies the existence of units of the same finished good (FG) in the same lot that differ in the aspect (quality), tone (color) and/or gage (thickness) [1,2] that should not be mixed to serve the same customer order. The usual consideration of three qualities, two

\footnotetext{
1 Complementary versions of this paper were presented in the "6th International Conference on Industrial Engineering and Industrial Management”, Vigo, July 2012, with the title "Managing qualities, tones and gages of Ceramic Supply Chains through Master Planning" and published in Informatica Economică, vol. 16, no. 3, pp.5-18, (2012) with the title "The Effect of Modeling Qualities, Tones and Gages in Ceramic Supply Chains' Master Planning”. The current paper provides significant additional content including a Decision Support System and additional results from different solution scenarios dealing with LHP uncertainty.
}

tones and three gages causes the existence of thirteen different subtypes of the same FG. This fact increases the volume of information and makes the ceramic system management more complex. Additionally, the customers from this type of companies tend to request quantities of different FGs in one same order, and they also require that the units of one same FG in the order are homogeneous.

LHP systems should face with a new kind of uncertainty [3]: the uncertainty in the future homogeneous quantities in production lots. Due to the inherent LHP uncertainty, the real homogeneous quantities of each subtype in a FG lot will not be known until their production was finished. Not knowing the homogeneous quantities available of the same FG to be promised to customers proves to be a problem when customers' orders have to be committed, reserved and served from homogeneous units available derived from the planned production. Furthermore, not accomplishing with this homogeneity requirement can lead to returns, product and company image deterioration, decreasing customer satisfaction and even lost of customers.

The order promising process (OPP) plays a crucial role in customer requirements satisfaction [3] and, also, in properly managing the special LHP characteristics. The OPP refers 
to the set of business activities that are triggered to provide a response to customer order requests [4]. This process requires information about available-to-promise (ATP) quantities, i. e. the stocks on hand or projected inflows of items stocked at the customer order decoupling point (already in transit or planned by the master plan) that has not yet been allocated to specific orders and thus can be promised to customers in the future. Because the master plan is a fundamental input to the OPP, one of the objectives and contributions of this paper is to define a master plan that considers LHP features and can provide this process with reliable information about future available homogeneous quantities.

Up to our knowledge there is no DSS that takes into account LHP features. Therefore, in this paper, we propose model-driven Decision Support System (DSS) for the operations planning of ceramic supply chains with diversity in qualities, tones and gages. Modeldriven DSS are designed so a user can manipulate model parameters to examine the sensitivity of outputs or to conduct a more ad hoc "what if?" analysis [5]. Thus, DSS functionalities are designed to allow the definition of several scenarios by changing input data, generating, evaluating and comparing different solutions through a series of interactive steps. Hence, dealing with assumptions is one of the main DSS roles [6]. Another important advantage of the DSS is that the Decision-Maker (DM) does not require understanding the complexities of the mathematical modeling, reducing the gap between theoretical contributions by researchers and the expectations of managers responsible for implementing the plans [7].

The system under our study can be considered as a Large Complex System (LSS). Filip and Leiviskä [8] indicate that LSS are characterized by their high dimensions (large number of variables), constraints in the information structure and the presence of uncertainties. The complexity of systems designed nowadays is mainly defined by the fact that computational power alone does not suffice to overcome all difficulties encountered in analyzing, planning and decision-making in presence of uncertainties. Thus, when human intervention is necessary, DSSs can represent a solution. These systems can help the decision-maker to overcome his/her limits and constraints he/she may face when approaching decision problems that count in the organization [9] and this is the objective of the DSS proposed in this paper.

The rest of the paper is structured as follows. Section 2 describes the problem under consideration and reviews the more closely related literature. Section 3 presents the mixed integer linear programming model proposed for the centralized master planning of ceramic SCs that explicitly takes into account LHP. Section 4 describes the DSS architecture. Section 5 shows the functionalities and practicability of the DSS through its application to a ceramic SC by means of realistic case. Finally, section 6 states the conclusions derived from the obtained results and future research lines.

\section{Problem Description}

In this paper, we consider the master planning problem for replenishment, production, and distribution in ceramic tiles SCs with LHP. These ceramic SCs are assumed to be multiitem, multi-supplier, multi-facility, multi-type and multi-level distribution centers. The characteristics of the problem under study are the same as in [10] but with relevant differences introduced by the LHP consideration [11] summarized in the following paragraph (Figure 1). As in [10] the Master Plan considers the Capacitated Lot-Sizing and Loading Problem with the aim of modeling the capacity consumption due to the high setup times among FGs and the fact that production lots of the same FG processed in different production lines present a high probability of not being homogeneous.

Furthermore, the splitting of each lot into homogeneous sub-lots of the same FG is also incorporated to reflect the LHP characteristics in a more realistic manner: different tones and gages for the first quality items. The sizing of lots for each production line is made in such a way that an integer number of customer order classes can be served from homogeneous quantities of each sub-lot. This aspect prevents a solution mixing quantities from different lots to serve a customer order, ensuring the homogeneity required by customers. To this end, different customer order classes are defined according to their size (Figure 1).

At the Master Plan level, demand forecasts are usually expressed in an aggregate manner without taking into account customer classes. Customer classes definition (also known as 


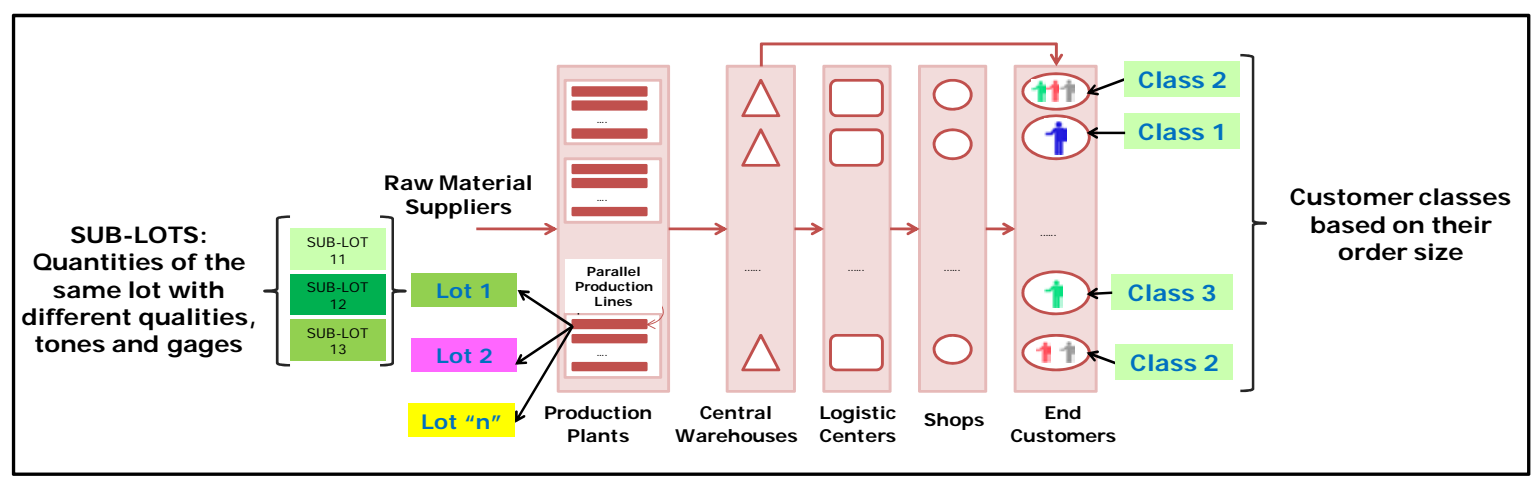

Figure 1. Main characteristics of Ceramic Supply Chains with LHP

customer segmentation) has been traditionally used in the field of the so called "allocation planning”. The allocation planning follows a push strategy (based on forecasts), as the master plan, but it is carried out after the master plan and before the OPP. The allocation planning has been used for improving the OPP results in shortage situations where demand is higher than supply quantities and the policy of promising orders in a first-come-first-served (FCFS) mode, entails the risk of promising scarce availabilities to the wrong customers; e.g., to less important customers or to customers with smaller profit margins [12]. During the allocation planning a classification scheme is defined that is used to segment and prioritize customer orders. For LHP contexts the homogeneous quantities manufactured should complete a whole FG's order size. For this reason, the classification attribute for defining customer classes is the order size.

Therefore, the consideration of customer classes for sizing lots and defining demand forecasts jointly with the splitting of lots into homogeneous sub-lots constitute the most relevant aspects that differentiate the model for master plan proposed in this paper from that proposed by Alemany et al. [10] and other models for SC master plan. The next section describes the mixed integer linear programming (MILP) model proposed to solve the described problem that constitutes the base for the DSS.

\section{The MILP Model for Master Planning of LHP Ceramic SCs}

To solve the ceramic SC master planning problem a mixed integer linear programming model (MP-CSC-LHP-1) is proposed. The model MP-RDSINC proposed by Alemany et al. [10] is considered as the starting point to formulate the present model but properly modified in order to reflect the LHP characteristics cited previously. The nomenclature (the indices, sets of indices, model parameters and decision variables) of the MP-CSC-LHP-1 model can be consulted on Tables 1 to 4, respectively, in [11]. The mathematical formulation is presented in the Annex. Those model elements that differ from the MP-RDSINC are written in italics.

For being concise, in this section only the MPCSC-LHP functions that differ from the MPRDSINC are described. For more details, the reader is referred to $[10,11]$. The objective function (1) expresses the gross margin maximization over the time periods that have been computed by subtracting total costs from total sales revenues. In this model, selling prices and other costs including the backlog costs can be defined for each customer class allowing reflect their relative priority.

Constraints (2) to (14) coincide with those of the MP-RDSINC and make reference to suppliers and productive limitations related to capacity and setup. Constraints (15)-(17) reflect the splitting of a specific lot into three homogeneous sub-lots of first quality $\left(\beta 1_{\text {ilp }}+\right.$ $\left.\beta 2_{\text {ilp }}+\beta 3_{\text {ilp }}=1\right)$. The number of sub-lots considered in each lot can be easily adapted to other number different from three. Through these constraints the sizing of lots is decided based on the number of orders from different customer order classes that can be served from each homogeneous sub-lot.

Customer order classes are defined based on the customer order size (i.e, the $\mathrm{m}^{2}$ ordered). Constraint (18) calculates for each time period, customer class and FG the total number of orders of a specific customer class that can be served from a certain lot by summing up the corresponding number of orders served by each homogeneous sub-lot of this lot. Constraint 
(19) derives the number of each customer order class that is possible to serve from the planned production of a specific plant. Through constraints (15-19), the production is adjusted not to the aggregate demand forecast as traditionally, but to different customer orders classes.

Furthermore, in contrast to the MP-RDSINC, the distributed, stocked and sold quantities downstream the production plants are expressed in terms of the customer class whose demand will be satisfied through them, being possible to discriminate the importance of each order class. Constraint (20) calculates the quantity of each FG to be transported from each production plant to each warehouse for each customer class based on the order number of each customer class that is satisfied by each production plant and the mean order size. Constraint (21) represents the inventory balance equation at warehouses for each finished good, customer class and time period. As backorders are permitted in both central warehouses and shops, sales may not coincide with the demand for a given time period. Backorder quantities in warehouses for each customer class are calculated using constraint (22). Constraint (23) limits these backorder quantities per customer class in each period in terms of a percentage of the demand of each time period. Constraint (24) forces to maintain a total inventory quantity higher or equal to the safety stock in warehouses. Constraint (25) is the limitation in the warehouses' capacity that is assumed to be shared by all the FG and customer order classes.

Constraint (26) represents the inflows and outflows of FGs and customer order classes through each logistic centre. Because it is not possible to maintain inventory in shops, constraint (27) ensures that the total input quantity of a FG for a specific customer class from warehouses to shops coincides with the quantity sold in shops. As backorders are permitted in both central warehouses and shops, sales may not coincide with the demand for a given time period. Constraints (28) and (29) are similar to constraints (22) and (23), respectively, but referred to shops instead of warehouses. The model also contemplates nonnegativity constraints and the definition of variables (30).

\section{The Model-Driven DSS}

The proposed Model-Driven DSS for the master planning of ceramic supply chains with LHP (DSS-LHP-CSC) meets the necessary requirements for DSS pointed out by Power and Sharda [5]:

- It uses different quantitative models. The DSS developed is based on the previous described model (MP-CSC-LHP-1) and another one (MP-CSC-LHP-2) defined to implement the different DSS functionalities.

- The designed model-driven DSS allows users manipulate model parameters through defining different scenarios in order to examine the sensitivity of outputs or to conduct a more ad hoc "what if?" analysis.

- It is accessible to a non-technical specialist in mathematical models

- It is designed to be used in a repetitive decision: the operations planning of ceramic supply chain is a period-driven decision.

The DSS-LHP-CSC architecture follows the generic dialog-data-modeling architecture proposed by Sprage [13]. The DSS building blocks include dialog, modeling and data components (Figure 2).

\section{Dialog components:}

- The user interface as the interaction point with the decision-maker. It is a combined graphical and tabular interface designed for providing a friendly interaction with the DSS.

- The user functionalities to provide the necessary interaction with the database and the models. The main DSS functionalities are: definition of scenarios, solve scenarios, robustness evaluation and compare solutions.

\section{Modeling components:}

- Models. The models are the main component in a Model Driven DSS. Two models (MPM-CSC-LHP-1 and MPMCSC-LHP-2) have been defined to support user functionalities.

- Solver. The Model Base Management System requires a solver found optimal solutions to the different models.

\section{Data components:}

- Data Base Management System (DBMS). It is in charge of the creation, access and update of data. 

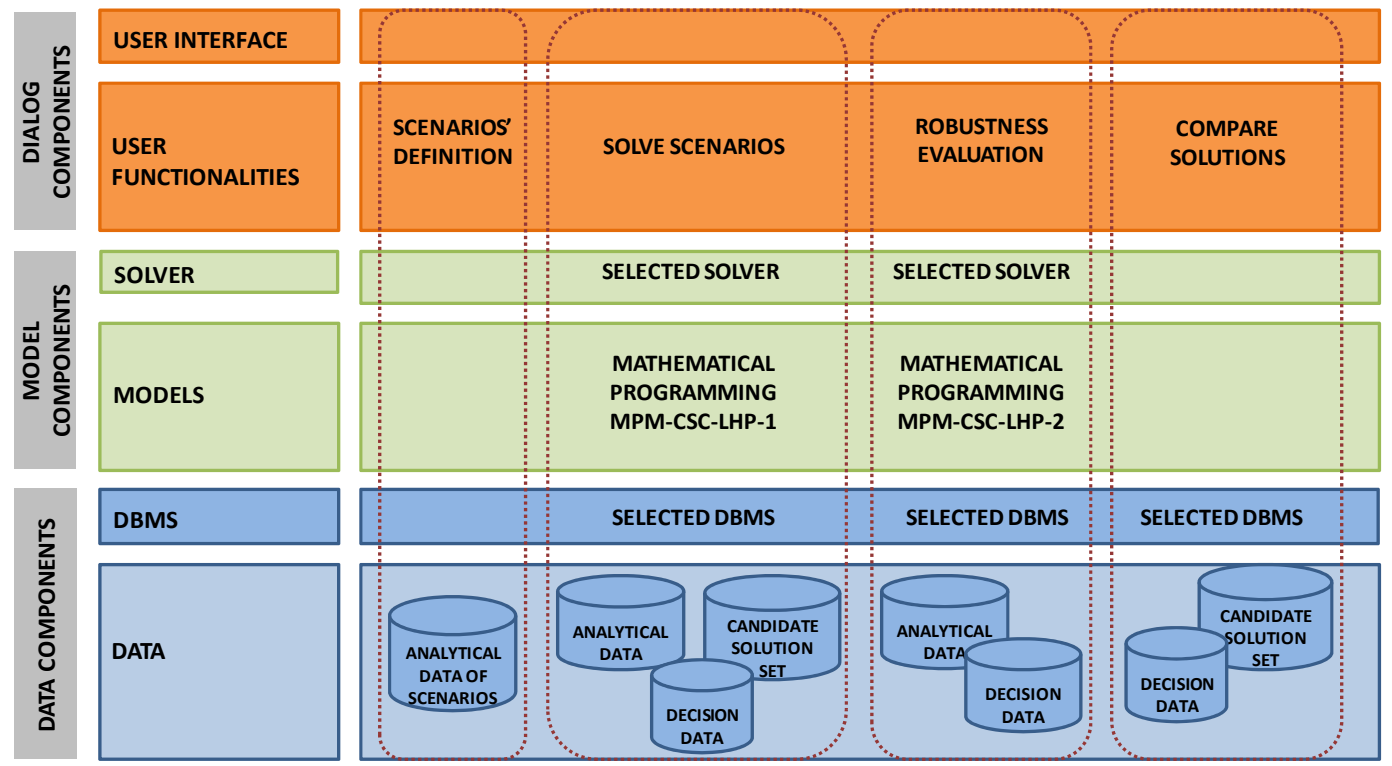

Figure 2. DSS-LHP-CSC Architecture

- Data. It is the collection of interrelated data organized to be use in the decision process. It includes Analytical Data as data required in the decision process, and Decision Data as information obtained in the decision process through the models' resolution.

Java v7 and the ECLIPSE platform have been used for developing the dialog components. MPL v4.2 has been selected to translate the mathematical programming models to a readable-machine format. GUROBI solver has been chosen due to the contrasted quality of solutions obtained. DB in Access has been used to store the corresponding data.

\section{Description of DSS-LHP-CSC Functionalities: an Illustration of a Ceramic Case}

We propose a DSS-LHP-CSC with a variety of functionalities that makes possible the DM to deal with assumptions in a friendly way. The DM could choose among different interactive options with the DSS and combine them in order to choose the final solution. During the process of finding the most satisfactory or optimal solution, the DM has the possibility of adding or removing solutions to the candidate solution set. The candidate solution set contains those solutions to the problem that are satisfactory and/or interesting for the DM and therefore, are candidate to be the finally chosen for being implemented.

With the aim of demonstrating the utility of the proposed DSS, an illustrative case has been developed which uses data derived from a real ceramic SC. The data for the case presented is the same used in [11] but with some modifications in order to illustrate the DSS functionalities that are described in the following.

Scenarios' Definition: this functionality (Figure 3) allows the DM to retrieve the necessary input data for obtaining the master plan through loading the corresponding data base (DB). The DM can define different scenarios retrieving data from different databases (Add DB). New scenarios can be also defined by means of copying and modifying one or more input data from a selected DB. The possible data to change is that regarding the objective function coefficients (profit/costs) and/or technological coefficients and/or righthand-side coefficients (times and capacities, demand, homogeneity parameters). The new scenarios can be saved in the set of scenarios. The DM can use this functionality for generating different situations for making "what-if" analysis as well as dealing with uncertainty in the data. The selected scenarios by the DM among those generated will be solved in the next functionality (Select to Solve). 
Because one of the distinguishing features of the proposed model is the LHP consideration, the proposed ceramic case tries showing how the DM can manage the inherent LHP uncertainty. For doing so, the DM will define different scenarios based on the value of beta parameters $\left(\beta 1_{i l p}, \beta 2_{i l p}, \beta 3_{i l p}\right)$. For the illustrative example (Figure 3), three scenarios have been defined using the beta coefficients. For the case under study, all three scenarios have been selected to solve. All scenarios assume that lots processed in different production lines and/or period of time are not homogeneous, but the degree of non uniformity in the units of the same production lot differs depending on the scenario:

- Optimistic scenario $\left(\beta 1_{i l p}=1, \quad \beta 2_{i l p}=0\right.$, $\beta 3_{\text {ilp }}=0$ ): This scenario assumes low heterogeneity, only one beta different from zero, meaning that units of the same production lot are all homogeneous.

- Probable scenario $\left(\beta 1_{i l p}=0.2, \quad \beta 2_{i l p}=0.8\right.$, $\beta 3_{\text {ilp }}=0$ ): This scenario assumes a medium heterogeneity, two betas different from zero, meaning that a production lot is divided into two homogeneous sub-lots.

- Pessimistic scenario $\left(\beta 1_{i l p}=0.1, \beta 2_{i l p}=0.4\right.$, $\left.\beta 3_{i l p}=0.5\right)$ : This scenario assumes high heterogeneity, three betas different from 0 , meaning that a production lot is divided into three homogeneous sub-lots.

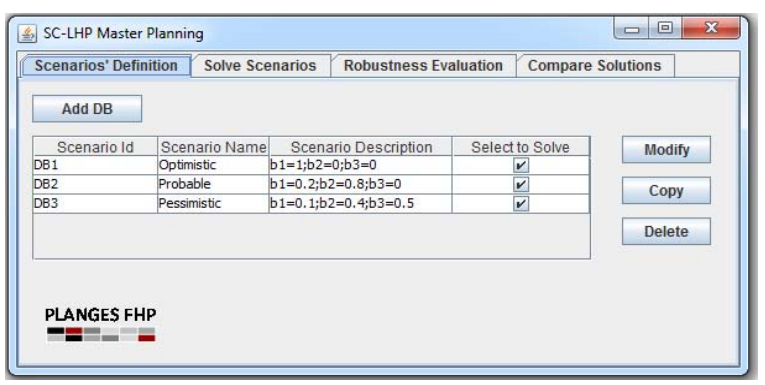

Figure 3. Definition of Scenarios

Solve scenarios: the selected scenarios in the previous functionality will be entered as input data for the MPM-CSC-LHP-1 that will be solved for each one of them. The DSS provides the value of the Objective Function of each solution and the gap for each set of data (scenario). The DM can make a deeper analysis of a selected solution through the "Detailed Solution" option. This detailed analysis allows the DM either view the value of the different components of the objective function (sales revenue, supply costs, production costs, setup costs, transportation costs, holding costs and backorder costs) and/or the decision variables.
As a result of this analysis the DM can eliminate solutions (Remove Solution) or select those satisfactory solutions to be incorporated to the candidate solution set (Save Solution).

For the example under consideration the optimal solution for the three scenarios appears in Figure 4. As it can be seen, the optimal solution to the optimistic scenario presents the maximum gross margin. After analyzing them, the DM can add interesting solutions to the candidate solution set. For the illustrative example, all the optimal solutions for each scenario have been selected to be added to the candidate solution set.

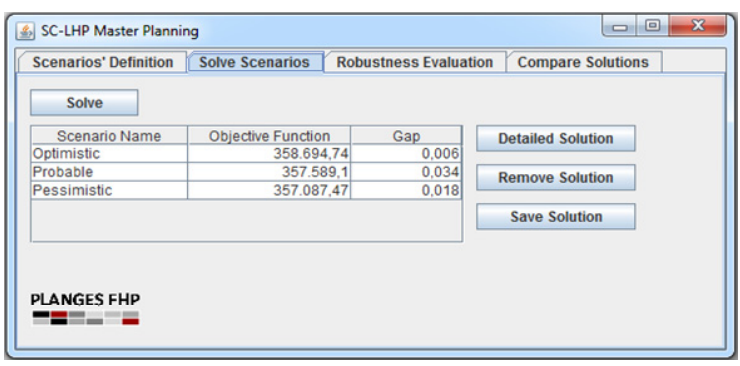

Figure 4. Resolution of Scenarios

Robustness Evaluation: It is important to highlight that the objective function value of the solution of each scenario would only be achieved if the solution implemented occurs in the corresponding scenario. Therefore, the DM should be interested in evaluating the behavior of the solutions generated in a specific scenario under other scenarios. For this, the DM should specify the solutions to be evaluated and the corresponding scenarios ("Select Solutions and Scenarios” option) (Figure 5). This functionality allows the DM to evaluate the robustness of the main decisions generated under a specific scenario when other situations occur.

In this case, our main decision is $M P_{\text {ilpt }}$ (amount of FG i manufactured on production line $\mathrm{l}$ of production plant $\mathrm{p}$ in period $\mathrm{t}$ ). Thus, a new version of the previously used MPM-CSCLHP-1 model has been defined (named MPMCSC-LHP-2).

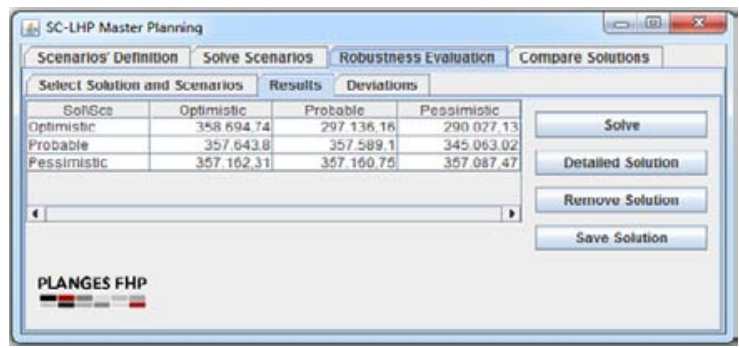

Figure 5. Robustness Evaluation of solutions under different scenarios 
This new model version considers the previous decision variable $\left(M P_{\text {ilpt }}\right)$ as a model parameter $\left(m p_{\text {ilpt }}\right)$ and simultaneously replaced the constraints (15-17) of the MPM-CSC-LHP-1 by the constraints (31-33). The original constraints (15-17) are relaxed from " =" to " $\geqslant$ " with the aim of ensuring a feasible solution, because with the specified $m p_{\text {ilpt }}$ obtained under a specific scenario (solution of the MPM-CSC-LHP-1) it will possible not to serve an integer number of customer orders under other scenarios.

$$
\begin{array}{ll}
\left(1-c m_{i}\right) * c q_{i} * \beta 1_{i l p} * m p_{i l p t} \geq \sum_{k} N K L 1_{i l p k t} * o r d q_{i k} & \forall p, \forall l \in L p(p), i \in I p(p), t k \\
\left(1-c m_{i}\right) * c q_{i} * \beta 2_{i l p} * m p_{i l p t} \geq \sum_{k} N K L 2_{i l p k t} * o r d q_{i k} & \forall p, \forall l \in L p(p), i \in I p(p), t k \\
\left(1-c m_{i}\right) * c q_{i} * \beta 3_{i l p} * m p_{i l p t} \geq \sum_{k} N K L 3_{i l p k t} * o r d q_{i k} & \forall p, \forall l \in L p(p), i \in I p(p), t k
\end{array}
$$

Through the "Solve" button of the Results Option (Figure 5), the execution of the new model MPM-CSC-LHP-2 is made for all selected solutions under the corresponding scenarios, providing the DM with the value of the objective function of a specific solution under other scenarios.

For the example under consideration, optimistic solution obtained as the optimal resolution of the model MPM-CSC-LHP-1 under the optimistic scenario is used to answer the following question: What happens if the DM implements the $M P_{\text {ilpt }}$ optimal solution obtained from the optimistic scenario $\left(m p_{\text {ilpt }}\right)$, but finally the probable or pessimistic scenario occurs? This question can be made for all solutions in the candidate solution list under all scenarios. Figure 5 provides the answer to this question for our case. The diagonal of the matrix, in this case, corresponds for the optimal solution under the corresponding scenario. The "Detailed Solution" option allows the objective terms analysis of solutions for each scenario. For our case, this study reveals that differences in the gross margin are mainly due to the backorder costs. Backorders exist for the optimistic solution in probable and pessimistic scenarios. Backorders also exist for the probable solution in pessimistic scenario. Finally, there are no backorders for the pessimistic solution under any scenario. From this analysis we can state that to consider the LHP in lots diminishes the gross margin (diagonal of the table in Figure 5), but provide more robust solutions under any scenario. For this analysis the DM can remove or save the solution for the candidate solution set.

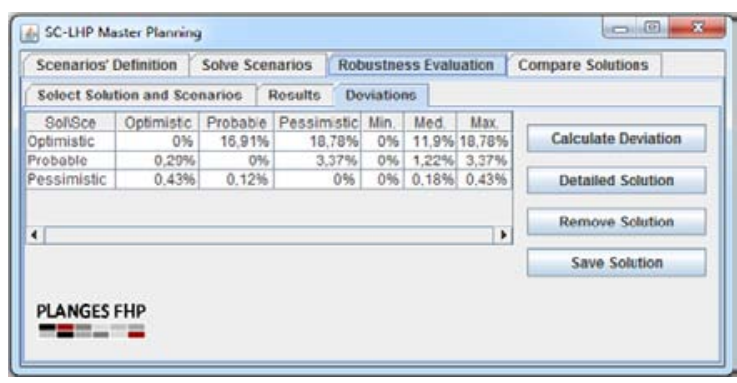

Figure 6. Relative performance of solutions under different scenarios

Compare solutions. By means functionalities 2 and 3, the DM can save and remove solutions from the candidate solution set. Through this functionality, at any moment of the decision process, the DM can select two different solutions from the candidate solution set and comparing them in terms of the global objective function or its components as well as in terms of the decision variables. As a result of this DSS functionality, the DM can remove some solutions from the candidate solution set. Finally, the DM should choose one solution of the candidate solution set as final solution, that is, as the final master plan to be implemented.

\section{Conclusions and Future Research}

This paper presents a mathematical programming model for the master planning of ceramic SCs characterized by LHP. Obtaining a satisfactory master plan in LHP industries involves dealing with a large number of variables and constraints in the information structure and the presence of uncertainties. Indeed, in LHP contexts appears a new source of inherent uncertainty: uncertainty in the 
quantities of homogeneous subsets of the same product available in planned production batches.

To facilitate the use of the mathematical programming model for practicing managers without the necessary mathematical knowledge, a model-driven DSS have been proposed. The DSS functional features are quite user-friendly and allow the DM to generate, analyze and compare different solutions. The DSS scenario definition capability constitutes a powerful tool to make what-if analysis, analyze the sensitivity of different operational and cost parameters and to deal with uncertainty in any input data of the model. The DSS utility have been shown by an illustrative realistic example of a ceramic SC where the definition of scenarios have been made based on the beta coefficients for representing the inherent LHP uncertainty. Furthermore, the DSS could be easily implemented in APS systems, reducing the gap of mathematical modeling power and its use by enterprises.

Future research lines include the consideration of a distributed and collaborative supply chain master planning process [14] among different SC's members. For this case, it could be very useful a DSS with a front-end web allowing reduce technological barriers and made it easier and less costly decision making for users in geographically distributed locations [15]. Furthermore, it will be very interesting to develop a web service that allows the company to do not be in charge of the solver and even of the model. This is because through this web service, the company could subcontract the necessary model modifications to fit it to their requirements.

The last future research line will be the integration of the proposed DSS with other order promising DSS for LHP contexts with the aim of providing reliable information about future uncommitted available homogeneous quantities (ATP-LHP). For those customer orders that cannot be committed with ATPLHP quantities it would be interesting to evaluate the possibility of defining new production lots using the uncommitted capacity (CTP) modifying, therefore, the initial master plan. These new research lines will allow a more flexible DSS to adapt the production to customer requirements and to face with discrepancies between plans and reality due to the inherent LHP uncertainty.

\section{Acknowledgements}

This research has been carried out in the framework of the project funded by the Spanish Ministry of Economy and Competitiveness (Ref. DPI2011-23597) and the Polytechnic University of Valencia (Ref. PAID-0611/1840) entitled "Methods and models for operations planning and order management in supply chains characterized by uncertainty in production due to the lack of product uniformity” (PLANGES-FHP)

\section{REFERENCES}

1. ALARCÓN, F., M. M. E ALEMANY, F. C. LARIO, R. F. OLTRA, La falta de homogeneidad del producto (FHP) en las empresas cerámicas y su impacto en la reasignación de inventario, Boletín de la Sociedad Española de Cerámica y Vidrio, vol. 50, no.1, 2008, pp. 49-58.

2. DAVOLI, G., S. A. GALLO, M. W. COLLINS, R. MELLONI, A Stochastic Simulation Approach for Production Scheduling and Investment Planning in the Tile Industry, International Journal of Engineering Science Technology, vol. 2, no. 9, 2010, pp 107-124.

3. ALEMANY, M. M. E., F. C. LARIO, A. ORTIZ, F. GOMEZ, Available-ToPromise Modeling for Multi-plant Manufacturing Characterized by Lack of Homogeneity in the Product: An Illustration of a Ceramic Case, Applied Mathematical Modelling, Available: http://dx.doi.org/10.1016/j.apm.2012.07.02 2 (in press).

4. ALEMANY, M. M. E., F. ALARCÓN, A. ORTIZ, F. C. LARIO, Order Promising Process for Extended Collaborative Selling Chain, Production Planning \& Control, vol. 19, no. 2, 2008, pp. 105-131.

5. POWER, D., R. SHARDA, Model-driven Decision Support Systems: Concepts and Research Directions, Decision Support Systems, vol. 43, 2007, pp. 1044-1061.

6. SEOK, H., S. Y. NOF, F. G. FILIP, Sustainability Decision Support System based on Collaborative Control Theory, Annual Reviews in Control, vol. 36, 2012, pp. 85-100. 
7. GOMES DA SILVA, C. G., J. FIGUEIRA, J. LISBOA, S. BARMAN, An Interactive Decision Support System for an Aggregate Production Planning Model based on Multiple Criteria Mixed Integer Linear Programming, Omega, vol. 34, 2006, pp. 167-177.

8. FILIP, F. G., K. LEIVISKÄ, Large-Scale Complex Systems, Part D, Automation Design: Theory and Methods for Integration. Springer Handbook of Automation, Springer, 2009, pp. 619-638.

9. FILIP, F. G., Decision Support and Control for Large-scale Complex Systems, Annual Reviews in Control, vol. 32, 2008, pp. 61-70.

10. ALEMANY, M. M. E., J. J. BOJ, J. MULA, F. C. LARIO, Mathematical Programming Model for Centralised Master Planning in Ceramic Tile Supply Chains, International Journal of Production Research, vol. 48, no. 17, 2010, pp. 5053-5074.

11. MUNDI, I., ALEMANY MME, BOZA, A., POLER, R., The Effect of Modeling
Qualities, Tones and Gages in Ceramic Supply Chains' Master Planning, Informatica Economică vol. 16, no. 3, 2012, pp.5-18.

12. MEYR, H., Customer Segmentation, Allocation Planning and Order Promising in Make-to-Stock Production, OR Spectrum, vol. 31, no. 1, 2009, pp. 229-256.

13. SPRAGUE, R. H., A Framework for the Development of Decision Support Systems, MIS Quarterly, vol. 4, no. 4, 1980, pp. 1-26.

14. POWER, D., S. KAPARTHI, Building Web-based Decision Support System, Studies in Informatics and Control, vol. 11, no. 4, 2002, pp. 291- 302.

15. ZARATÉ, P., Decision Making Process: A collaborative Perspective, Studies in Informatics and Control, vol. 17, no. 2, 2008, pp. 225-230.

Annex (MP-CSC-LHP-1 mathematical formulation)

\section{Objective Function:}

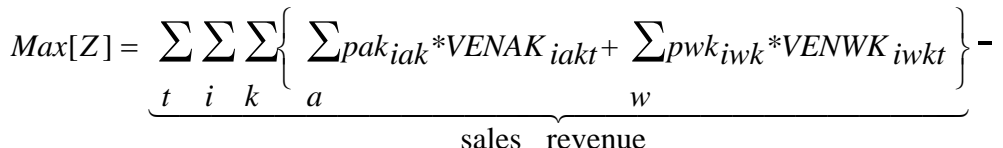

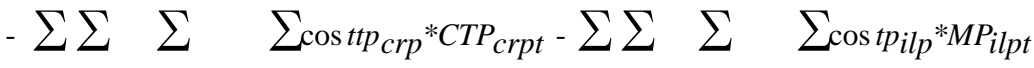

$$
\begin{aligned}
& \underbrace{t \quad p \quad \mathrm{r} \in \mathrm{Rp}(\mathrm{p}) \mathrm{c} \in \mathrm{Cr}(\mathrm{r})}_{\text {supply costs }} \underbrace{t \quad p \quad \mathrm{l} \in \mathrm{Lp}(\mathrm{p}) \mathrm{i} \in \mathrm{Il}(\mathrm{l})}_{\text {production costs }} \\
& -\sum \sum \sum \sum \text { costsetup flp }{ }^{*} Z_{F} \text { flpt }-\sum \sum \sum \sum \text { costsetupi } i_{i l p} Z^{*} I_{\text {ilpt }} \\
& \begin{aligned}
t \quad p \mathrm{l} \in \mathrm{Lp}(\mathrm{p}) \mathrm{f} \in \mathrm{Fl}(\mathrm{l}) \quad t \quad p \mathrm{l} \in \mathrm{Lp}(\mathrm{p}) \mathrm{i} \in \mathrm{Il}(\mathrm{l}) \\
\text { setup }
\end{aligned}
\end{aligned}
$$

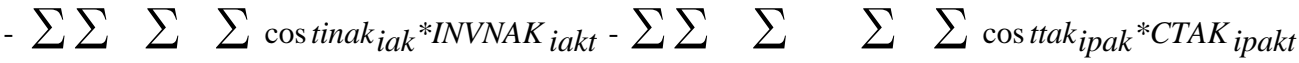

$$
\begin{aligned}
& \underbrace{t \quad a \mathrm{i} \in \mathrm{Ia}(\mathrm{a}) k}_{\text {holding costs }} \underbrace{t \quad a \mathrm{p} \in \mathrm{Pa}(\mathrm{a}) \mathrm{i} \in \operatorname{Ip}(\mathrm{p}) k}_{\text {transport costs }}
\end{aligned}
$$

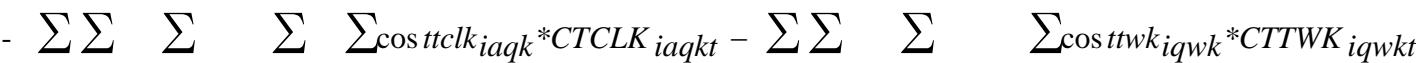

$$
\begin{aligned}
& \begin{array}{ll}
t & a \quad \mathrm{q} \in \mathrm{Qa}(\mathrm{a}) \mathrm{i} \in \operatorname{Iq}(\mathrm{q}) k \quad t \quad q \quad \mathrm{w} \in \mathrm{Wq}(\mathrm{q}) \mathrm{i} \in \operatorname{Iw}(\mathrm{w})
\end{array} \\
& \text { transport costs } \\
& \text { - } \sum \sum \sum \sum_{\text {costdifak }} \text { iak }^{*}{ }^{*} \text { IFAK }_{\text {iakt }}-\sum \sum \sum \sum_{\text {costdifwk }} \text { iwk }^{*}{ }^{*} \text { DIFWK }_{\text {iwkt }} \\
& t \quad a \mathrm{i} \in \mathrm{Ia}(\mathrm{a}) k \\
& t \quad w \mathrm{i} \in \operatorname{Iw}(\mathrm{w}) k
\end{aligned}
$$




\section{Constraints:}

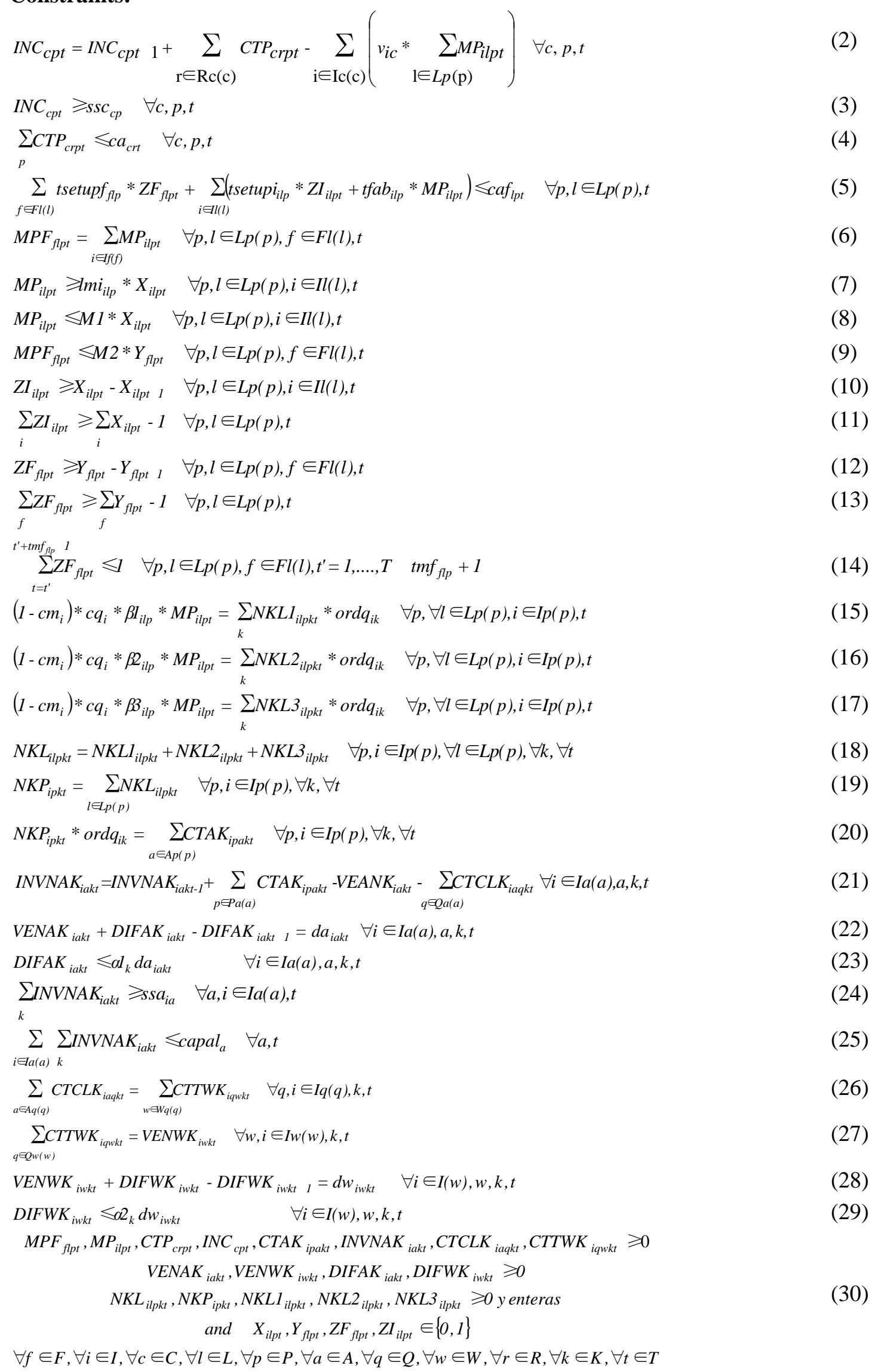

